Dr. Roberto Gelado-Marcos

Universidad CEU San Pablo

@ roberto.geladomarcos@ceu.es iD 0000-0002-4387-5347

Dra. Belén Puebla-Martínez

Universidad Rey Juan Carlos

@ belen.puebla@uric.es

(iD 0000-0002-1481-4238
- Recibido / Received

12 de junio de 2020

- Aceptado / Acepted 29 de junio de 2020

- Páginas / Pages De la 239 a la 252

- ISSN: $1885-365 \mathrm{X}$

\title{
La relación entre conciencia regional y relevancia mediática. Un análisis de la representación de las comunidades autónomas en los cibermedios españoles
}

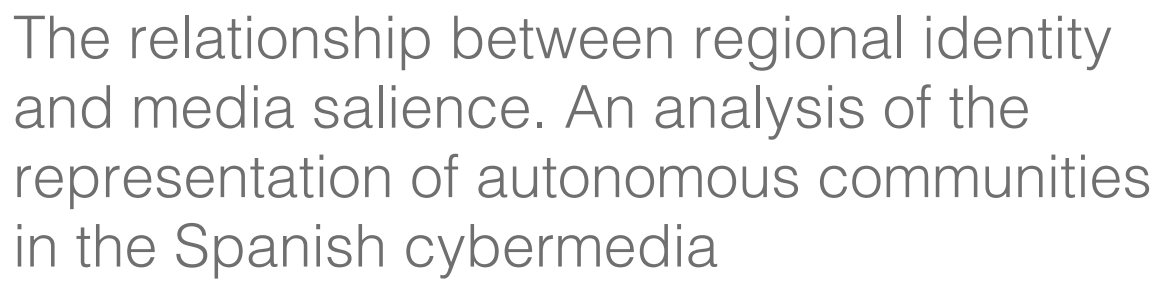

\section{RESUMEN:}

El papel de los cibermedios en la configuración de la opinión pública es un fenómeno que, lejos de ser reciente, no ha parado de consolidarse desde los albores del nuevo siglo. La presente investigación aborda la correlación entre los sentimientos regionales de los españoles y la representación en cibermedios de las Comunidades Autónomas como elemento representativo de la importancia de los medios digitales en la determinación del framing asignado a temas de relevancia de la esfera pública. Para ello se ponen en relación, por un lado, y a través de tres indicadores de análisis, la presencia de las Comunidades Autónomas españolas en la prensa digital de referencia y, por el otro, la expresión de sentimientos regionalistas asociados a las distintas comunidades. Los resultados arrojan un claro desnivel en la cobertura hacia unos y otros territorios, con un claro predominio de tres Comunidades Autónomas, Madrid, Cataluña y País Vasco, un bloque intermedio configurado por Andalucía y Comunidad Valenciana, y un tercer bloque con el resto de las Comunidades. La cobertura mediática del primer grupo triplica al del segundo y sextuplica a las Comunidades del tercer grupo, lo que certifica la hipótesis de que la conciencia regional exacerbada es correlativa a una mayor cobertura mediática de las Comunidades que la tienen y que aquellas que no tienen una conciencia regional tan exacerbada, por lo general, suelen tener menos cobertura mediática. Está investigación ha sido realizada gracias a la financiación obtenida por la Junta de Castilla y León y el Fondo Social Europeo. 


\section{ABSTRACT:}

The role played by cybermedia in setting up the public opinion is a phenomenon that, far from being recent, has continued to consolidate from the beginning of the new century. This research approaches the correlation between the regional awareness of the Spanish population and the representation of the autonomous communities in the digital press as a representative element of how important digital media are in framing relevant aspects of the public sphere. In order to do so, this paper links, on the one hand, and through three indicators, the media salience of the Spanish autonomous communities and, on the other, the regional awareness associated to the different regions. The results show a clear gap in the media coverage, with a clear predominance of three autonomous communities, Madrid, Catalonia and the Basque Country, an intermediate block configured by Andalusia and the Valencian Community, and a third block with the rest of the communities. The media coverage of the first group triples that of the second and sextuples the communities of the third group, which confirms the hypothesis that the exacerbated regional awareness is correlated to a greater media coverage and that those communities with a less exacerbated regional awareness generally tends to have less media coverage. This research has been carried out thanks to the financing obtained by the Junta de Castilla y León and the European Social Fund.

KEY WORDS:

Media representation; Internet; Digital media; Autonomous communities; Regional identities.

\section{Introducción y objetivos}

La prevalencia de la cobertura mediática de ciertas Comunidades Autónomas españolas sobre otras cuya representación es inferior en número y variedad de temas cubiertos es un fenómeno sobre cuya actualidad han llamado la atención autores como Liñeira (2014, p. 149), quien recientemente constataba una distinta cobertura informativa en las comunidades españolas. Centrándose más concretamente en el reciente auge de la pulsión secesionista en las esferas de poder catalanas Juarez Miro (2020) ha señalado también el papel de los medios de comunicación para comprender mejor los sucesos acaecidos en Cataluña en los últimos meses. La presente investigación acepta estos enfoques, pero defiende que, en realidad, la dispar representación de las Comunidades Autónomas en los medios nacionales, con el efecto que esto tiene en la visibilidad de las regiones y su consiguiente percepción de importancia no es, ni mucho menos, reciente. El presente estudio se plantea comprobar la vigencia de este fenómeno en el pasado reciente y trata de demostrar que la hiperrepresentación de ciertas Comunidades Autónomas es no solo un fenómeno de fondo, sino también transversal a los principales medios españoles.

El papel de los medios de comunicación como definidores primarios de los elementos que circulan en la esfera pública es algo que, además de ocupar durante cerca de un siglo a los investigadores de la comunicación de masas, ha cobrado especial vigencia con la consolidación de Internet y los cibermedios como actores principales en la escena mediática. Desde la aparición de la World Wide Web a finales de los años 80, la expansión de Internet como plataforma indispensable para explicar los fenómenos de influencia mediática solo ha ido en ascenso. La presente investigación trata, por un lado, de apuntalar esta convicción de la influencia de los cibermedios en la configuración de la opinión pública en aspectos cruciales de la política y la sociedad actuales y, por otro, de recordar que, lejos de ser un fenómeno novedoso, es posible encontrar casos en un pasado reciente que certifican una cierta longevidad de este fenómeno de influencia a través de los cibermedios.

En un escenario político actual, el español, que parece haber devuelto el debate recurrente sobre las identidades regionales y la configuración autonómica (Navarro Ardoy, Ruiz 
Jiménez, Ferri Fuentevilla, 2017; Tajadura Tejada, 2019), la contribución de los medios a este debate se antoja esencial en la configuración de la esfera pública. Por ello, la presente investigación plantea, además, la pertinencia de volver la vista atrás unos años para entender los términos de esta contribución; en concreto, para tratar de averiguar si las comunidades autónomas con una repercusión mediática más exacerbada han recibido una mayor repercusión mediática en los cibermedios españoles de referencia.

Para ello, el estudio se centró en el comienzo de la década de 2010; sobre la base, por un lado, de que Internet ya era una plataforma de referencia, como se demuestra en la figura 1 y, por otro, de que ya por entonces la representación de las comunidades autónomas en los cibermedios permite vislumbrar ideas interesantes para el análisis de la situación actual de este debate en la esfera pública española.

El porqué de la elección de la prensa digital frente a otros medios de comunicación arraigados previamente a otros soportes como la televisión tiene su justificación también en el tipo de consumidor de cibermedios, cuya absorción de medios tradicionales difiere sensiblemente al observado en el conjunto de la población. «Leen más diarios, suplementos, dominicales y revistas, pero ven menos televisión, lo que se justifica por su elevado nivel educativo y su edad», explican Sádaba y Portilla, (2005, p. 117). Esta tendencia, que establece una proporción directa entre el menor consumo de televisión para quienes consumen más Internet, también ha sido confirmada en Estados Unidos a través de los estudios realizados por Nie (2004).

Es interesante, además, observar la progresión que está experimentando la prensa digital en los últimos años con respecto a su homóloga en papel. La encuesta AIMC a usuarios de Internet 2010 ya arrojaba datos de interés en este sentido que son especialmente pertinentes para nuestro estudio de caso:

- Internet era la fuente principal de información de actualidad para dos tercios de los encuestados (AIMC, 2011).

\section{El consumo de medios en Internet}

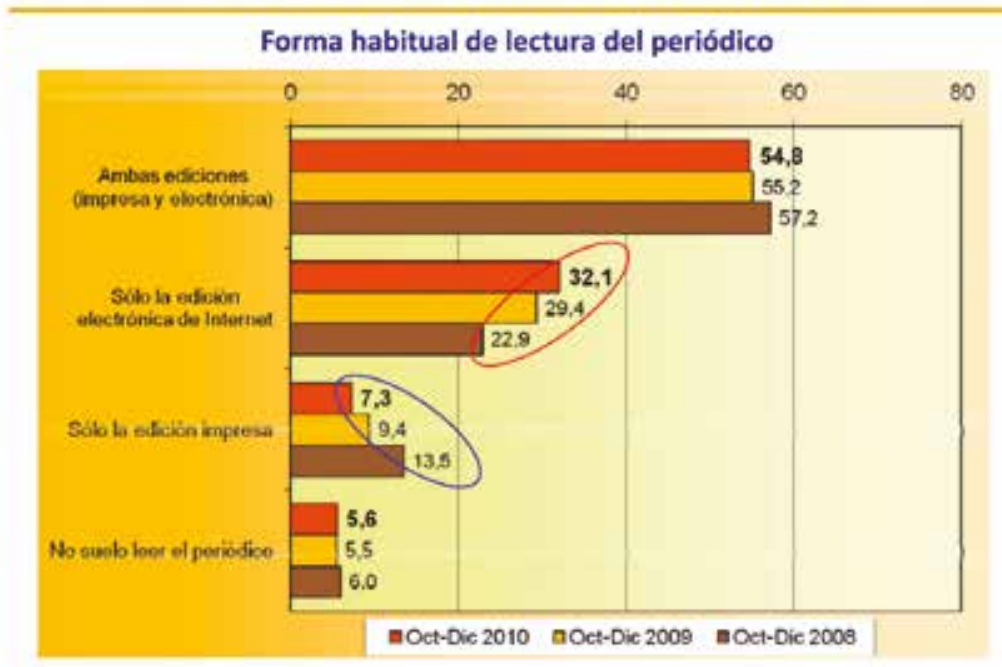

Figura 1. El consumo de medios en Internet. Fuente: AIMC. 
- La lectura de los diarios electrónicos era ya una práctica cada vez más cotidiana: un $78 \%$ de los encuestados por la AIMC (2011) manifestó haber leído algún periódico digital en el día anterior, lo que significa un incremento porcentual de 5 puntos con respecto a la última oleada. Los datos contrastan con el descenso de un $5 \%$ de los encuestados que manifestaron haber leído diarios en papel el día anterior (un $46 \%$ ).

- Aunque siguen predominando los lectores simultáneos de ambos formatos (un 55\%), lo cierto es que, de entre los que se decantan sólo por una de las dos versiones prevalecen claramente los que se informan únicamente a través de la prensa digital (32\%) frente a los que siguen prefiriendo el formato tradicional impreso (7\%) (AIMC, 2011).

El Observatorio Nacional de las Telecomunicaciones y de la Sociedad de la Información (ONTSI) para Eurostat incidía también en la imparable emergencia de lo digital en los últimos meses. Según estos datos, el $40 \%$ de los españoles emplea la Red para leer periódicos o revistas digitales, lo cual sitúa a España ligeramente por debajo de Reino Unido (43\%) y Alemania (42\%), pero muy por encima de la media europea (34\%) y de países como de Francia (21\%), Italia (24\%), Portugal (29\%) o Grecia (25\%).

Todas estas tendencias no hacen sino reafirmar la apuesta inicial por la prensa digital en el presente estudio. Por un lado vemos que el perfil de los consumidores de cibermedios se asemeja bastante al de ciertas elites, si no de poder, sí de intelectuales que pueden convertirse fácilmente en líderes de opinión. López-García et al. (2005a) añaden, además, una característica diferencial de lo cibernético frente a lo impreso: la distinción entre cibermedios nacionales, provinciales, regionales o locales se difumina por el carácter deslocalizado y la «ruptura de las barreras geográficas» que posibilita el medio electrónico. De este mapa también resulta interesante, por lo que de significativo tienen estos datos (aunque hayan de ser tomados con la perspectiva del tiempo) en el refrendo de ciertas hipótesis barajadas en el presente trabajo de investigación. Según las investigaciones de López-García et al. (2005a), en España había 1.274 cibermedios hacia la mitad de la década de 2000. De ellos, 761 (el $59,73 \%$ ) eran cibermedios de información generalista. Pues bien, 143 (casi uno de cada cinco) eran catalanes, frente a 96 madrileños, 71 vascos y 69 andaluces.

\section{Definición de objetivos y metodología}

El objetivo principal de la presente investigación se centra en determinar si existe una correlación significativa entre la repercusión mediática y el sentimiento identitario de las distintas comunidades autónomas. A raíz de ese objetivo planteado, se formuló la siguiente hipótesis: Las comunidades con mayor conciencia regional tienen más repercusión mediática en la prensa nacional.

La hipótesis requiere, para su constatación o rechazo, definir dos variables principales: la repercusión mediática y la conciencia regional. Se entiende como repercusión mediática el volumen de noticias publicadas sobre una comunidad autónoma. Para medir la repercusión mediática se emplearon tres baremos principales (noticias, módulos y módulos ponderados), que descansan sobre los principios del análisis de contenido cuantitativo y los fundamentos del diseño periodístico mencionados anteriormente, y que se explicarán con detalle más adelante. 
La segunda variable, «conciencia regional», podría definirse como el grado de apego a la región por exaltación de la identidad propia o por oposición a la identidad española. Para su medición, se decidió tomar los datos de la pregunta número 20 del Barómetro Autonómico II de 2010 realizado por el INE, en la que se preguntaba a los encuestados qué significaba España para ellos, ya que es la única de las estudiadas que combina tanto el elemento de orgullo regional como la posibilidad de rechazo a una entidad española por encima de su región. Como vimos, las respuestas se podían simplificar en tres grandes apartados, "Conciencia regional exacerbada», "Conciencia regional moderada» $y$ «Sin conciencia regional», lo cual nos proporcionaba los datos necesarios para medir esta variable.

A modo de refuerzo, aunque siempre tomando como referencia las respuestas a esta pregunta número 20 del Barómetro Autonómico II, también se consideraron como elementos de medición de la variable «Conciencia regional» los datos correspondientes a las respuestas a las preguntas 21 (sobre el orgullo de ser español) y 22 (sobre el orgullo regional), así como la encuesta, también del Barómetro Autonómico, sobre la popularidad de las opciones políticas nacionalistas en las distintas comunidades.

Para establecer correlaciones se utilizó de manera predominante el coeficiente de correlación de Pearson y, de forma secundaria, el coeficiente Rho de Spearman. El coeficiente de Pearson puede emplearse para medir el grado de relación de dos variables si ambas utilizan una escala de medida de intervalo/razón (variables cuantitativas), como es el caso para la variable «nivel de repercusión mediática» y conciencia regional. El resultado de las correlaciones según este tipo estadístico fluctúa entre dos valores numéricos, +1 y -1 ; de modo que, cuanto más se acerque el resultado a cualquiera de los extremos, mayor será la correlación (directa o inversa, respectivamente). Después de consultar a varios expertos en estadística y diversas fuentes bibliográficas de referencia en la materia, se certificó que el primer umbral de significatividad en las correlaciones se establecería en +/-0,3.

\section{Diseño de la investigación}

El análisis de la prensa digital se centró en los medios escritos de ámbito nacional. Esto implica no sólo medios de comunicación de difusión nacional, sino esencialmente generalistas. López-García et al. definían cibermedio de información generalista:

aquel emisor de contenidos referentes a las diversas áreas del saber humano que tiene voluntad de mediación entre hechos y público, utiliza fundamentalmente criterios y técnicas periodísticas, usa el lenguaje multimedia, es interactivo e hipertextual, se actualiza y se publica en la red Internet (López-García et al., 2005a, p. 62).

Algunas de esas nociones, en especial la multimedialidad y la hipertextualidad, se recogieron como elementos potenciadores de la visibilidad de las piezas analizadas.

En su análisis comparativo de cibermedios, López-García et al. eligieron cuatro criterios: que fueran «diarios de calidad, líderes en el mercado de la información general y con un ámbito de difusión nacional», además de contar «con una edición consolidada en Internet» (López-García et al., 2005b, pp. 127-132). Ponderando los datos de audiencia, raigambre histórica del medio y proyección generalista, optamos por enfocar nuestro estudio a dos cibermedios de referencia, elmundo.es y elpais.com. 
Con vistas a certificar la premisa explicada en el capítulo introductorio de la vigencia nada reciente de los cibermedios en el ecosistema mediático y su influencia en temas tan relevantes de la esfera pública, se escogió como año de estudio el 2010. En el caso concreto de la prensa digital, el número total de noticias recolectadas fue de 6.475 , con lo cual haciendo una estimación similar al alza tendríamos un universo aproximado de 50.000 noticias. Con un universo de 50.000 noticias y una muestra de 6.475 , considerando $p$ y q para el peor de los casos posibles $(p=0,5$ y $q=0,5)$, con un nivel de confianza del $99 \%$, se calculó que el error muestral general que depararía la muestra de prensa impresa con respecto a un universo total era de $\pm 0,015$.

Dadas las características de la prensa digital y los estudios de legibilidad empleados, así como las secciones compartidas por los distintos medios sometidos a estudio, se consideró que el análisis debía centrarse tanto en las portadas como en las secciones de España, Economía, Tecnología, Cultura y Sociedad. La recopilación del material analizado contaba, además, con la peculiaridad de que, en el caso de elpais.com, el archivo digital no conservaba las características de la portada del día, sino que se disponían los titulares y pequeños blurbs con formato de flujo de texto (aglutinados en las diferentes secciones).

\subsection{Medición de la presencia en la prensa digital}

Para la medición de la unidad de análisis «presencia» en medios digitales se optó por adaptar el Método de Análisis Proporcional Aproximativo (MAPA) propuesto por Tena Parera (1997) a la pantalla de ordenador. Si el MAPA propugnaba una división modular de la página de periódico en diez líneas horizontales y diez líneas verticales, lo que daba lugar a cien cuadros formato (divididos, a su vez, en una nueva rejilla de 10×10), se empleó una plantilla similar para medir la presencia en las portadas del formato digital. Un segundo criterio cuantitativo empleado para la medición de la presencia se centró no tanto en la cantidad de espacio concedido a las Comunidades Autónomas como en el número de noticias publicadas relativas a cada una de ellas.

\subsection{Medición de la presencia matizada en la prensa digital}

La medición de la unidad de análisis «presencia matizada» requería de un enfoque investigador más cualitativo que el de la «presencia». Dado que el archivo de elmundo.es conservaba tres versiones de la portada diaria del cibermedio, mañana, tarde y noche, se optó por elegir la segunda sobre la base de los datos de consumo a diferentes horas del día. Definido esto, se llegó a la conclusión de que, como ya se vio en la descripción de las características diferenciales de la producción en cibermedios, los productos ideados para este soporte necesitaban un análisis que tuviera en cuenta la posición en la página, la multimedialidad o la hipertextualidad, por citar algunos. Por todo ello, se adoptó el modelo de análisis desarrollado por Gelado Marcos, Magro Vela y Rubira (2018).

\section{Resultados}

En los resultados comparados del número de noticias totales publicadas en prensa digital, observamos que tanto en elpais.com como en elmundo.es se invierten los resultados de las dos primeras posiciones, tal y como se observa en la tabla 1. 
Tabla 1. Datos comparativos de prensa digital por Comunidad y número total de noticias publicadas

\begin{tabular}{|c|c|c|c|c|}
\hline Posición & Comunidad Autónoma & elmundo.es & Comunidad Autónoma & elpais.com \\
\hline 1 & Madrid & 611 & Cataluña & 1.036 \\
\hline 2 & Cataluña & 420 & Madrid & 940 \\
\hline 3 & Andalucía & 267 & Comunidad Valenciana & 597 \\
\hline 4 & País Vasco & 226 & Andalucía & 417 \\
\hline 5 & Comunidad Valenciana & 204 & País Vasco & 374 \\
\hline 6 & Castilla y León & 99 & Castilla y León & 110 \\
\hline 7 & Galicia & 89 & Galicia & 104 \\
\hline 8 & Castilla La Mancha & 74 & Castilla La Mancha & 100 \\
\hline 9 & Canarias & 56 & Cantabria & 99 \\
\hline 10 & Asturias & 51 & Aragón & 79 \\
\hline 11 & Baleares & 50 & Asturias & 48 \\
\hline 12 & Aragón & 49 & Canarias & 45 \\
\hline 13 & Extremadura & 39 & Baleares & 39 \\
\hline 14 & Cantabria & 36 & Región de Murcia & 38 \\
\hline 15 & Región de Murcia & 29 & La Rioja & 33 \\
\hline 16 & Ciudades Autónomas & 23 & Navarra & 28 \\
\hline 17 & Navarra & 20 & Extremadura & 24 \\
\hline 18 & La Rioja & 2 & Ciudades Autónomas & 19 \\
\hline \multicolumn{2}{|l|}{ TOTAL } & 2.345 & & 7.529 \\
\hline
\end{tabular}

Fuente: Elaboración propia.

Sin embargo, mientras en elpais.com se mantiene más o menos el patrón de cobertura similar en Madrid y Cataluña (en torno a un $10 \%$ más de noticias publicadas sobre esta segunda comunidad), en el caso de elmundo.es sí se aprecia un vuelco reseñable: no sólo es Madrid y no Cataluña la comunidad con mayor cobertura por número de noticias totales, sino que la distancia se eleva hasta más del $30 \%$ entre una y otra (611 noticias publicadas sobre Madrid, frente a las 420 de Cataluña). Andalucía, País Vasco y Comunidad Valenciana, tal y como ocurría en la edición impresa, copan las tres siguientes posiciones, separadas ya por un margen significativo de las tres primeras.

La brecha entre estas tres comunidades que ocupan las posiciones de la $3^{\mathrm{a}}$ a la $5^{\mathrm{a}}$ con el resto de las autonomías es, igualmente, significativa. Este tercer vagón aparece encabezado en las versiones digitales de El Mundo y El País por Castilla y León, que cuenta con una proporción de impacto mediático por número de noticias totales de 1 a 6 en el caso de elmundo.es y de 1 a 10 en el caso de elpais.com. 
Igualmente destacable, en este caso por su invisibilidad al foco mediático, es el caso de La Rioja en elmundo.es, ya que durante todo el periodo sometido a estudio tan sólo se han recogido dos noticias referidas a esta autonomía.

Tal y como se indicó en el apartado metodológico, a fin de realizar una medición más exacta de la cobertura mediática que se concedía a las diferentes comunidades autónomas durante el año sometido a estudio se realizó el cálculo no sólo del número de noticias sino del número de módulos dedicados a cada autonomía.

Se computó así, el número de módulos en la portada de cada una de las secciones; si bien en el caso de elpais.com, como se advirtió antes también, esto resultó imposible ya que el archivo de noticias aparece en forma de flujo y no refleja la misma disposición en pantalla que tuvieron las noticias en el momento de su publicación. Por ello, los datos de espacio en módulos referidos a prensa digital sólo se computaron en el caso de la versión digital de El Mundo. Siendo así las cosas, los resultados totales del espacio en módulos publicado sobre cada autonomía se pueden observar en la tabla 2.

Tabla 2. Datos comparativos por Comunidad y número de módulos publicados

\begin{tabular}{|l|c|}
\hline \multicolumn{1}{|c|}{ Comunidad Autónoma } & Número de módulos \\
\hline Cataluña & $27.364,64$ \\
\hline Madrid & $26.950,1$ \\
\hline País Vasco & $18.640,3$ \\
\hline Comunidad Valenciana & $8.478,3$ \\
\hline Andalucía & $8.389,5$ \\
\hline Castilla y León & $3.927,8$ \\
\hline Castilla La Mancha & $3.779,1$ \\
\hline Galicia & $3.416,9$ \\
\hline Cantabria & $1.981,4$ \\
\hline Aragón & $1.845,3$ \\
\hline Canarias & $1.789,2$ \\
\hline Asturias & $1.741,9$ \\
\hline Baleares & 1.289 \\
\hline Extremadura & $1.287,8$ \\
\hline Región de Murcia & $1.175,9$ \\
\hline Navarra & 679,3 \\
\hline Ciudades Autónomas (Ceuta y Melilla) & 618,4 \\
\hline La Rioja & 111,9 \\
\hline
\end{tabular}

Fuente: Elaboración propia. 
Como vemos, los resultados en cuanto a número de módulos repiten las mismas tendencias observadas anteriormente. Por un lado, Cataluña y Madrid vuelven a copar las dos primeras posiciones, con escasas diferencias entre una y otra y una gran brecha entre las dos y sus inmediatas perseguidoras.

Castilla y León es la primera de las comunidades que viven, por así decirlo, alejadas del foco mediático. En este caso, encabeza un grupo de tres, compuesto por Castilla La Mancha, Galicia y ella misma, cuya cobertura mediática rebasa los tres mil módulos, situándose proporcionalmente en la mitad aproximadamente de Andalucía y Comunidad Valenciana, algo menos de un cuarto de la cobertura del País Vasco y menos de un sexto del número de módulos que reciben Cataluña y Madrid en los medios sometidos a estudio.

El tercer elemento de medida, denominado «módulos ponderados» a lo largo del proceso de investigación, introducía un coeficiente corrector resultante de la aplicación de múltiples factores dependientes del diseño que ya se explicaron en el apartado metodológico. Como también se anticipó antes, por las características del archivo digital de elpais.com resultó imposible aplicar este coeficiente corrector a este medio (ya que no se conservaban elementos mensurables como la ubicación en página, el tamaño de la noticia, etc.). En cambio, el coeficiente corrector en función de las peculiaridades del diseño periodístico sí se aplicó a las noticias extraídas de elmundo.es.

Los resultados observados en la tabla 3 ratifican las tendencias vistas en los indicadores anteriores: Madrid y Cataluña lideran la atención mediática, muy por encima porcentualmente de sus tres inmediatas seguidoras - de nuevo, Cataluña, País Vasco y Andalucía. Sin embargo, las diferencias en términos absolutos entre Castilla y León y las comunidades que figuran por debajo de ella en la lista no son muy grandes (por más que en términos relativos puedan ser significativas). Al igual que se comentó antes, sí son mayores estas diferencias cuando se mira a las regiones que figuran por encima de Castilla y León: por cada módulo ponderado de Castilla y León se publican dos sobre el País Vasco (una relación similar a la que se mantiene con Andalucía y Comunidad Valenciana), casi cuatro sobre Cataluña y casi seis sobre la Comunidad Autónoma de Madrid. Por todo ello, se constata de nuevo un flujo informativo más o menos constante para 12 comunidades, con brechas significativas a medida que se va ascendiendo desde la quinta posición hasta la primera de la tabla.

Una vez analizados los datos de publicación por comunidades y secciones en los medios sometidos a estudio, se resolvió explorar las posibles relaciones existentes entre tales estadísticas y otras variables relevantes desde el punto de vista autonómico. Así, se consultaron diferentes variables que el Instituto Nacional de Estadística actualiza con relativa frecuencia para dilucidar cuáles podrían dar mejor respuesta a las subhipótesis planteadas en la metodología. Fruto de esta búsqueda se estimó pertinente estudiar, en primer lugar, la relación existente entre los sentimientos de apego a las diferentes regiones o autonomías y el nivel de repercusión mediática de cada una de estas regiones o autonomías.

En el análisis comparado de los índices de repercusión mediática y el sentimiento de pertenencia a las comunidades autónomas se observa que existe una relación directa y bastante significativa entre el volumen de producción mediática referida a una región y la exaltación de su sentimiento identitario, tal y como se puede comprobar en la figura 2. 
Tabla 3. Datos totales por Comunidad y módulos ponderados en elmundo.es

\begin{tabular}{|l|c|}
\hline \multicolumn{1}{|c|}{ Comunidad Autónoma } & Módulos ponderados \\
\hline Madrid & $8.384,1$ \\
\hline Cataluña & $5.409,97$ \\
\hline País Vasco & $3.079,25$ \\
\hline Andalucía & $2.797,75$ \\
\hline Comunidad Valenciana & $2.685,9$ \\
\hline Castilla y León & $1.460,3$ \\
\hline Galicia & $1.131,7$ \\
\hline Castilla La Mancha & 922,3 \\
\hline Asturias & 822,65 \\
\hline Canarias & 592,1 \\
\hline Aragón & 559,35 \\
\hline Baleares & 525,3 \\
\hline Extremadura & 476,65 \\
\hline Cantabria & 392,3 \\
\hline Navarra & 309,1 \\
\hline Región de Murcia & 292,9 \\
\hline Ciudades Autónomas (Ceuta y Melilla) & 272,95 \\
\hline La Rioja & 100,8 \\
\hline & \\
\hline
\end{tabular}

Fuente: Elaboración propia.

Como se puede observar en las tablas 4 y 5 , las correlaciones no sólo son significativas, sino que además cumplen la regla de ser directas en el caso de que las distintas variables de producción mediática se pongan en relación con los indicadores del Barómetro Autonómico 2010 que resaltan la conciencia regional, e inversas con aquellas respuestas de los encuestados que no resaltan la conciencia regional.

Por todo ello, queda demostrada la correlación que se planteaba en la hipótesis sobre la relación entre la conciencia regional y el nivel de repercusión mediática de las comunidades autónomas en España en 2010. Como conclusión general, se puede afirmar que aquellas comunidades con mayor sentimiento regionalista (ya sea expresado de manera positiva, como exaltación de una conciencia regional exacerbada o expresión de una filiación política nacionalista, o negativa, como oposición a la pertenencia a España) gozan de una mayor repercusión mediática en los periódicos sometidos a estudio. 


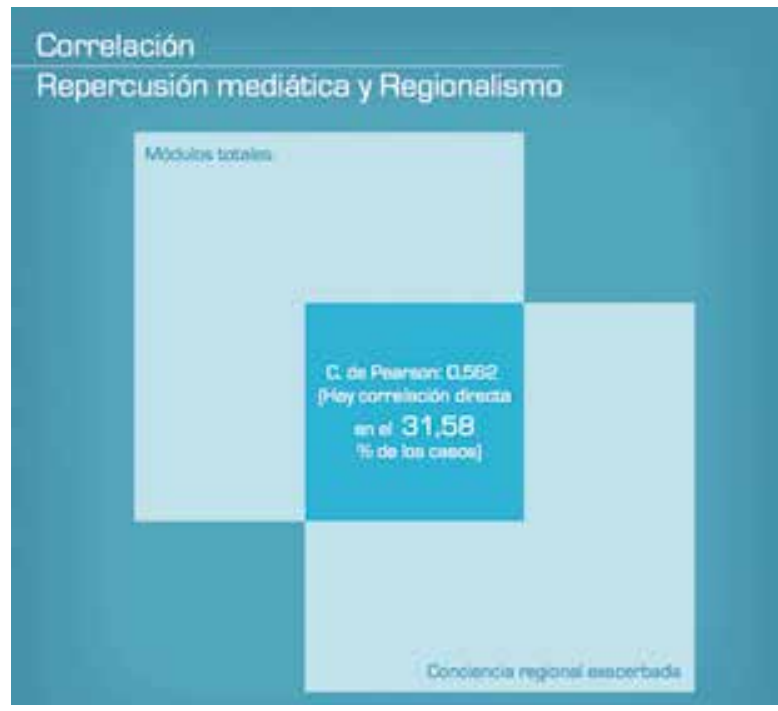

Figura 2. Correlación entre la repercusión mediática y sentimiento regional. Fuente: elaboración propia.

Tabla 4. Correlación entre la repercusión mediática y regionalismo (I).

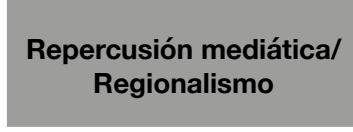

Noticias totales

Módulos totales

Módulos ponderados totales

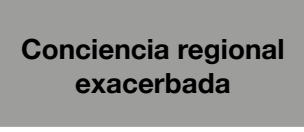

$$
0,402
$$

0,562

0,503

\begin{tabular}{|c|}
\hline $\begin{array}{c}\text { Poco o nada } \\
\text { orgulloso de ser } \\
\text { español }\end{array}$ \\
\hline 0,459 \\
\hline 0,582 \\
\hline 0,55 \\
\hline
\end{tabular}

\begin{tabular}{|c|}
\hline $\begin{array}{c}\text { Muy o bastante } \\
\text { orgulloso de ser } \\
\text { español }\end{array}$ \\
\hline 0,587 \\
\hline 0,532 \\
\hline 0,58 \\
\hline
\end{tabular}

Fuente: Elaboración propia/Barómetro Autonómico II (INE).

Tabla 5. Correlación entre la repercusión mediática y regionalismo (II).

\begin{tabular}{|l|c|c|c|}
\hline $\begin{array}{c}\text { Repercusión mediática / } \\
\text { Regionalismo }\end{array}$ & $\begin{array}{c}\text { Sin conciencia } \\
\text { regional }\end{array}$ & $\begin{array}{c}\text { Muy o bastante } \\
\text { orgulloso de ser } \\
\text { español }\end{array}$ & $\begin{array}{c}\text { Poco o nada } \\
\text { orgulloso de ser } \\
\text { de la comunidad }\end{array}$ \\
\hline Noticias totales & $-0,351$ & $-0,454$ & $-0,663$ \\
\hline Módulos totales & $-0,487$ & $-0,57$ & $-0,542$ \\
\hline Módulos ponderados totales & $-0,457$ & $-0,553$ & $-0,672$ \\
\hline
\end{tabular}

Fuente: Elaboración propia/Barómetro Autonómico II (INE).

\section{Conclusiones/Discusión}

La investigación planteada ha confirmado la hipótesis sugerida respecto a la correlación entre el retrato mediático de las comunidades autónomas y los cibermedios españoles. El análisis 
de la cobertura periodística de 2010 demuestra que existe una elite informativa copada por Madrid y Cataluña, seguida de un grupo de tres regiones compuesto por País Vasco, Comunidad Valenciana y Andalucía que se lleva una buena porción del pastel informativo, aunque comparativamente mucho menor a la de sus predecesoras en la lista; y un último grupo de doce comunidades que parecen vivir más alejadas del foco mediático de la prensa nacional.

Castilla y León es la más importante de las comunidades menos importantes a ojos de los medios, a juzgar por la cobertura que de ella hacen las versiones digitales de El Mundo y El País. Sin embargo, la diferencia absoluta entre el tercer vagón y los dos primeros es mucho más pronunciada que la que existe entre las doce comunidades de cola.

La medición ponderada de la cobertura mediática establece también tres grandes grupos, si bien compuestos por diferentes comunidades. El primero engloba a tres comunidades, Cataluña, Madrid y País Vasco, que se mueven en cifras parecidas. El segundo está integrado por la Comunidad Valenciana y Andalucía, a las que las tres comunidades que ocupan el vagón de cabeza triplican en cobertura mediática ponderada. Y el último, encabezado por Castilla y León, engloba a las doce comunidades restantes, que apenas alcanzan la mitad de la cobertura del grupo intermedio y no reciben más que una sexta parte de la atención mediática de Cataluña, Madrid y País Vasco.

La correlación entre los datos sobre exaltación de la conciencia regional, obtenidos a partir de los Barómetros Autonómicos del CIS, y los datos de cobertura mediática de las comunidades autónomas, obtenidos a partir del estudio de caso de esta tesis, revelan que existe una vinculación significativa entre ambas variables. Eso nos permite verificar la hipótesis de que aquellas comunidades con mayor sentimiento regionalista (ya sea expresado de manera positiva, como exaltación de una conciencia regional exacerbada o expresión de una filiación política nacionalista, o negativa, como oposición a la pertenencia a España) gozan de una mayor repercusión mediática.

Una vez se ha demostrado que el retrato mediático de las comunidades autónomas refleja un claro desequilibrio cuantitativo entre las distintas regiones españolas, se puede afirmar que los medios están contribuyendo, sea o no de manera pretendida, a acentuar la brecha entre comunidades autónomas españolas. Sea o no intencional, los medios responden al discurso de diferenciación alimentado durante años desde distintas esferas políticas. Noelle-Neumann (1995) ya había hablado de la consonancia, la acumulación, la omnipresencia y el clima de opinión como factores cruciales para entender el funcionamiento de la opinión pública, con lo que la teoría de la espiral del silencio sirve de respaldo a nuestra formulación anterior: que a base de producir, de manera más o menos uniforme y a través de distintos medios y soportes, un retrato desequilibrado de las distintas regiones españolas lo que se consigue es multiplicar el desequilibrio.

También se podría aplicar a la expresión misma del nacionalismo la tesis del silenciamiento de las opiniones para evitar el aislamiento. Si los individuos tienden a percibir que el nacionalismo excluyente es la expresión predominante de la opinión pública, puede suceder que quienes no tengan esta interpretación excluyente de las identidades silencien su opinión para evitar el rechazo social.

Que exista una infrarrepresentación de ciertas comunidades en el discurso periodístico español significa en los términos de McCombs y Shaw (1972) que se está sacando a estas regiones de la agenda mediática y, por ende, en buena medida de la agenda pública porque, 
como decía Cohen (1963), puede que los medios no puedan decirle a su audiencia durante mucho tiempo qué tienen que pensar, pero sí pueden ser tremendamente influyentes a la hora de determinar sobre qué tienen que pensar (cf. también lyengar y Kinder, 1993). La presencia sobredimensionada de unas comunidades o el silenciamiento de los temas relacionados con otras es, pues, una forma, sea pretendida o no, de ahondar en la brecha interautonómica y la exaltación de los hechos diferenciales.

De esta manera, los resultados del presente estudio se ven respaldados por las dos teorías predominantes en la actualidad en cuanto al fenómeno de la opinión pública, la agenda-setting y la espiral del silencio, que tienen una aplicación inmediata en la presente investigación.

\section{Bibliografía}

AIMC (2011). Encuesta AIMC a usuarios de Internet 2010. 25 de febrero de 2011.

COHEN, Bernard C. (1963). The press and foreign policy. Princeton (NJ): Princeton University Press.

GELADO MARCOS, Roberto, MAGRO-VELA, Silvia, y RUBIRA, Rainer (2018). «Ponderación de la importancia asignada a las noticias en los medios escritos. Propuesta de un análisis en función de los elementos del diseño periodístico». En Rodríguez Rodríguez Jorge Miguel, López Pan Fernando y Albalad Aiguabella, José María (Coords.). Calidad informativa y nuevas narrativas. Madrid: Egregius.

IYENGAR, Shanto y KINDER, Donald R. (1993). La televisión y la opinión pública: la información es poder, Gernika: Mexico DF.

JUÁREZ MIRO, Clara (2020). Identity discourses about Spain and Catalonia in news media: Understanding modern secessionism. London: Lexington Books.

LIÑEIRA, Robert (2014). El Estado de las autonomías en la opinión pública: preferencias, conocimiento y voto. Madrid: Centro de Investigaciones Sociológicas

LÓPEZ-GARCÍA, Xosé, LIMIA FERNÁNDEZ, Moisés, ISASI VARELA, Antonio, PEREIRA FARIÑA, Xosé, GAGO MARIÑO, Manuel y CALVO DIÉGUEZ, Rosario (2005a). «Tipología de los cibermedios». En Salaverría, Ramón (Coord.). Cibermedios. El impacto de internet en los medios de comunicación en España. Sevilla: Comunicación Social, pp. 39-70.

LÓPEZ-GARCÍA, Xosé, LIMIA FERNÁNDEZ, Moisés, ISASI VARELA, Antonio, PEREIRA FARIÑA, Xosé, GAGO MARIÑO, Manuel y CALVO DIÉGUEZ, Rosario (2005b). «Análisis comparativo de cibermedios: Lavanguardia.es, Elmundo.es y Elpaís.es». En Salaverría, Ramón (Coord.). Cibermedios. El impacto de internet en los medios de comunicación en España. Sevilla: Comunicación Social, pp. 125-143.

McCOMBS, Maxwell y SHAW, Donald L. (1972). «The agenda setting function of mass media». Public Opinion Quarterly, 36, pp. 176-187. DOI: 10.1086/267990

NAVARRO ARDOY, Luis, RUIZ JIMÉNEZ, Antonia María y FERRI FUENTEVILLA, Elena (2017). «La organización territorial española en crisis. Análisis del pensamiento político de las élites locales y regionales en los partidos de ámbito estatal de izquierda». Revista Española de Sociología, 26(1), 53-68. DOI:10.22325/fes/res.2016.3

NIE, N. H. (2004). «Ten years after the birth of Internet, how do Americans use the Internet in their daily lives?». Stanford Center for the Quantitative study of Society, Diciembre. 
NOELLE-NEUMANN, Elisabeth (1995). La espiral del silencio. Opinión pública: nuestra piel social. Barcelona: Paidós.

SÁDABA, Charo y PORTILLA, Idoia (2005). «Modelos de negocio». En Salaverría, Ramón (Coord.). Cibermedios. El impacto de internet en los medios de comunicación en España. Sevilla: Comunicación Social, pp. 82-121.

TAJADURA TEJADA, Javier (2019). «El futuro de las provincias y las diputaciones provinciales ante una reforma de la constitución territorial». Teoría y Realidad Constitucional, 43, 229-256. DOI: 10.5944/ trc.43.2019.24409

TENA PARERA, Daniel (1997). La influencia de la composición gráfica en la elección de un bloque de texto escrito. Tesis doctoral dirigida por Ángel Rodríguez Bravo y presentada en el Departamento de Comunicación Audiovisual y Publicidad de la Universidad Autónoma de Barcelona. Recuperado de https://ddd.uab.cat/record/37388 